${ }^{1}$ Department of Surgery, McMaster Paediatric Eye Research Group, McMaster University, Hamilton, Ontario, Canada

${ }^{2}$ Department of Surgery, McMaster Pediatric Surgery Research Collaborative, McMaster University, Hamilton, Ontario, Canada

${ }^{3}$ Ophthalmology Clinic, McMaster Children's Hospital, Hamilton Health Sciences, Hamilton, Ontario, Canada

${ }^{4}$ Department of Pediatric Ophthalmology, Stollery Children's Hospital, Edmonton, Alberta, Canada

${ }^{5}$ Department of Ophthalmology, University of Alberta, Edmonton, Alberta, Canada

${ }^{6}$ Orthoptic Clinic, Department of Ophthalmology, BC Children's Hospital, Vancouver, British

Columbia, Canada

Correspondence: K Sabri, Ophthalmology Clinic, McMaster Children's Hospital, Hamilton Health Sciences, 1200 Main St W, 3V2, Hamilton, Ontario L8N 3Z5, Canada

Tel: +1905 5212100 x73509;

Fax: +1 9055708968.

E-mail: sabrik@mcmaster.ca

Received: 8 January 2016 Accepted in revised form:

12 September 2016

Published online:

4 November 2016

\section{Elbow splinting as a method to increase patching compliance in amblyopia therapy}

\author{
Abstract \\ Purpose The purpose of the study was to \\ evaluate the feasibility and acceptability of \\ utilizing elbow splints as a method of \\ increasing amblyopic patching compliance in \\ pediatric ophthalmology patients who fail to \\ comply with prescribed patching regimens. \\ Patients and methods Patients $<6$ years of \\ age who were not adhering to pre-specified \\ patching guidelines were prescribed elbow \\ splints in order to increase patching \\ compliance at the discretion of a pediatric \\ ophthalmologist. If the child was non- \\ compliant (patching $<50 \%$ of specified time \\ or consistently removing patch), parents were \\ asked to try using the elbow splints until \\ patching compliance was achieved. Non- \\ parametric Wilcoxon signed-rank tests were \\ used to compare patching time pre- and post- \\ splints. \\ Results $41 / 48$ children who were prescribed \\ elbow splints to increase patching \\ compliance for amblyopia were included for \\ analysis. Seven children were excluded due \\ to patching and splints being prescribed at \\ the outset $(n=4)$, and for not using the \\ splinting intervention $(n=3)$. Median age at \\ being prescribed elbow splints was 37 \\ (12-68) months. Mean daily patching prior to \\ splints was $1.5 \pm 1.7 \mathrm{~h}$, whereas mean \\ prescribed daily patching was $4.95 \pm 1.5 \mathrm{~h}$. \\ Following splinting, 34/41 (83\%) became \\ compliant with patch alone, and visual \\ acuity increased in 39/41 (95\%) patients, with \\ no patients developing amblyopia in the \\ fellow eye. Median amount of time splints \\ was required to improve compliance was 7 \\ (1-240) days. \\ Conclusion Elbow splinting seems to be a \\ viable alternative method to increase pediatric \\ amblyopic patching compliance when \\ patching alone fails to achieve satisfactory \\ compliance. \\ Eye (2017) 31, 406-410; doi:10.1038/eye.2016.222; \\ published online 4 November 2016
}

K Sabri ${ }^{1,2,3}$, B Easterbrook², B Wakeman ${ }^{4,5}$,

$\checkmark$ Mehta $^{6}$ and R Riyaz ${ }^{5}$

Introduction

Amblyopia is the reduction in best corrected visual acuity (VA) of an eye without the presence of an organic cause ${ }^{1}$ and the importance of its treatment in early childhood cannot be overstated. Good eyesight is a key component to physical, emotional, and social development. Not only do individuals with uncorrected amblyopia have triple the risk of depression, double the risk of mortality, and greater difficulties with day-to-day living ${ }^{2}$ but they have more than twice the risk of non-amblyopic individuals for losing vision in their good eye and becoming visually impaired in both eyes for the remainder of their lives. $^{3}$

There are a variety of different treatment modalities for amblyopia, but occlusion therapy, commonly known as patching, is the most frequently utilized, and is the cornerstone of most successful amblyopia treatment strategies. ${ }^{4,5}$ Patching is an economically feasible therapy to improve VA in the affected eye, though this therapy relies entirely on patient compliance for effectiveness.

Unfortunately, compliance rates have been shown to average $<50 \%$ for a variety of reasons including skin irritation, poor cosmesis, and lengthy treatment periods. ${ }^{5}$ Although anecdotal evidence exists of using elbow splints ${ }^{6,7}$ to increase occlusion therapy compliance, to the best of our knowledge, the study presented is the largest of its kind assessing the use of elbow restraints for improving compliance with patching for amblyopia.

The aim of this study was to assess the efficacy, safety, and parental acceptance of using elbow restraints as a tool to improve occlusion therapy compliance in children with amblyopia. 


\section{Materials and methods}

\section{Participants}

After receiving Research Ethics Board approval, a retrospective review was conducted of the medical records of amblyopic children admitted to two tertiary pediatric ophthalmology centers in Canada, who were fitted for elbow splints between January 2008 and December 2011. Inclusion criteria were as follows: children ( $<6$ years old) diagnosed with amblyopia by an ophthalmologist, who had attempted patching therapy for a minimum of 3 months and who had been fitted for elbow splints due to low treatment adherence.

\section{Intervention}

Whether or not children in clinic were prescribed elbow splints as an adjunct compliance measure for amblyopia therapy was at the discretion of the pediatric ophthalmologist. Parents/guardians were offered elbow splinting as an adjunct treatment option if their child was considered to be non-compliant with the patching regimen they had been prescribed. For the purpose of this study, non-compliance was defined as patching $<50 \%$ of the specified amount of time, or consistently removing the patch during daily activities. Parents/guardians were instructed to continue to attempt patching the nonamblyopic eye for the predetermined amount of time, and to employ the elbow splints if the child removed the patch, until the child was fully compliant with daily patching. Ultimately, parental discretion determined if one or both arms were splinted, as well as when patching and/or utilization of the elbow splints stopped, though they were provided with instructions to continue use until the child was fully compliant.

\section{Data collection}

Data abstraction was conducted by one of the authors. Data fields collected included sex, age, cause of amblyopia, hours asked to patch prior to splinting, hours patched prior to splinting, pre- and post-VA, hours patched following splinting, follow-up duration, and parental opinions of the patch and splints. Parental opinions of elbow splints were routinely collected in clinic using self-report Likert Scales and, therefore, accessible through chart review. Hours patched pre- and postsplinting were obtained through parental reporting. Descriptive statistics were summarized using means, medians, quartiles, and ranges and all data explored for trends to assess normality. Non-parametric Wilcoxon signed-rank tests were used to compare splinting time with a significance level of 0.05 . All statistical analysis was conducted in SPSS. ${ }^{8}$

\section{Results}

Of the 48 patients who received elbow splints at the two tertiary-care centers, 41 patients were included (16 females, 25 males) in the study for analysis, with a median age of 37 (12-68) months at the time of being prescribed elbow splints. Four patients were excluded from analysis due to being prescribed splints from the outset of patching therapy because of distance from the ophthalmologist, and three were excluded for being prescribed splints but not using the intervention (Figure 1). Mean follow-up duration was $4.9 \pm 1.6$ months. Regarding etiology, 24 (59\%) participants presented with Esotropia as the primary cause of amblyopia, 15 (37\%) with Anisometropia, and 2 (4\%) with congenital cataracts.

Among the 41 children, the mean amount of daily patching prescribed prior to the use of elbow splints was $4.95 \pm 1.5 \mathrm{~h}$, whereas mean amount of actual patching achieved prior to the use of splints was only $1.5 \pm 1.7 \mathrm{~h}$. On average, children were patching $3.5 \mathrm{~h}$ less a day than was recommended by the ophthalmologist to improve VA in the amblyopic eye. Following the use of elbow splints as a compliance measure, the mean amount of patching achieved without splints increased to from $1.5 \pm 1.7 \mathrm{~h}$ to $3.4 \pm 1.3 \mathrm{~h}$. VA measurements in the amblyopic eye increased in 39/41 (95\%) children following elbow splinting, while two study participants maintained the VA measured prior to splinting (Table 1). Age and sex were not statistically significant predictors of

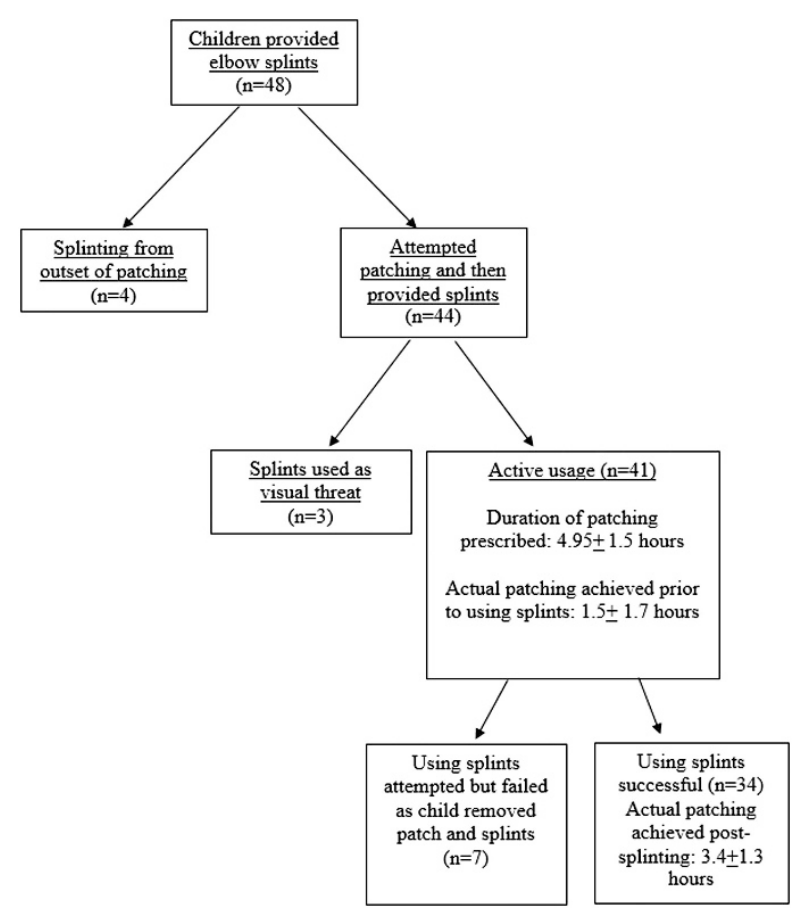

Figure 1 Flow diagram of study design. 
Table 1 Visual acuity in affected eye

\begin{tabular}{lcc}
\hline Visual acuity measurements & $\begin{array}{c}\text { Pre-splinting } \mathrm{n} \\
(\%)\end{array}$ & $\begin{array}{c}\text { Post-splinting } \mathrm{n} \\
(\%)\end{array}$ \\
\hline $20 / 30$ & $0(0)$ & $1(3)$ \\
$20 / 40$ & $0(0)$ & $7(17)$ \\
$20 / 50$ & $3(7)$ & $4(10)$ \\
$20 / 60$ & $0(0)$ & $3(7)$ \\
$20 / 70$ & $3(7)$ & $2(5)$ \\
$20 / 80$ & $9(22)$ & $6(15)$ \\
$20 / 100$ & $6(15)$ & $1(3)$ \\
$20 / 200$ & $3(7)$ & $0(0)$ \\
$<1 / 60$ & $3(7)$ & $0(0)$ \\
$1 / 60$ & $0(0)$ & $3(7)$ \\
Fumbles 3 mm bead & $1(3)$ & $0(0)$ \\
Locates 3 mm bead & $0(0)$ & $1(3)$ \\
Fumbles 1 mm bead & $3(7)$ & $0(0)$ \\
Locates 1 mm bead & $0(0)$ & $3(7)$ \\
Uncentral, steady, & $9(22)$ & $0(0)$ \\
unmaintained & & \\
Central, steady, & $1(3)$ & $10(23)$ \\
unmaintained & & \\
Total & $41(100)$ & $41(100)$ \\
\hline
\end{tabular}

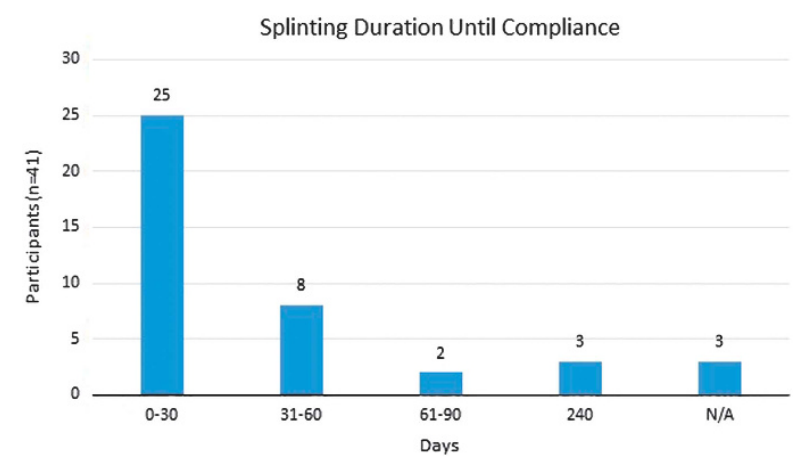

Figure 2 Splinting duration until compliance.

splinting success in this cohort of patients. Also of note, is that none of the children developed amblyopia in the fellow eye following the use of elbow splints to increase patching compliance.

After elbow splinting was introduced, 34/41 children (83\%) became compliant with wearing the patch alone without continuously using the splints as well. Seven children (17\%) remained non-compliant with patching after splinting was initiated, as the majority ( $5 / 7$ children) were able to remove the splints and then the patch.

Among the 41 children who were no longer using splints at the time of the questionnaire, the median (range) amount of time the splints were used to modify behavior and improve patching compliance was 7 (1-240) days (Figure 2).

When asked what they would recommend for another child who was non-compliant with patching
Table 2 Parental opinions regarding occlusion therapy $(N=41)$

\begin{tabular}{|c|c|}
\hline Question asked of parents & Response \\
\hline \multicolumn{2}{|c|}{$\begin{array}{l}\text { Would you recommend elbow splints for another child in the same } \\
\text { scenario? }\end{array}$} \\
\hline Yes $(\%)$ & $34(83)$ \\
\hline No $(\%)$ & 7 (17) \\
\hline \multicolumn{2}{|c|}{ What was your initial reaction when splints were suggested? $\mathrm{n}(\%)$} \\
\hline Strongly opposed & $0(0)$ \\
\hline Opposed & $9(22)$ \\
\hline Neutral & $13(32)$ \\
\hline In favor & $6(14)$ \\
\hline Strongly in favor & $13(32)$ \\
\hline \multicolumn{2}{|c|}{$\begin{array}{l}\text { How did your child react when the splints were put on for the first time } \\
\text { without the patch? Median }\left(Q 1^{a}, Q 3^{b}\right)\end{array}$} \\
\hline $1=$ strongly upset, $5=$ neutral or happy & $1(1,4)$ \\
\hline \multicolumn{2}{|c|}{$\begin{array}{l}\text { How did your child react to the combination of splints and patch? } \\
\text { Median }\left(Q 1^{a}, Q 3^{b}\right)\end{array}$} \\
\hline $1=$ strongly upset, $5=$ neutral or happy & $1(1,2)$ \\
\hline
\end{tabular}

${ }^{a}$ First quartile: middle number between the smallest and median numbers within the data. ${ }^{b}$ Third quartile: middle number between the median and highest number within the data.

therapy to treat amblyopia, 34 (83\%) parents/guardians said that they would recommend using the elbow splints as a method to increase patching compliance (Table 2).

A non-parametric Wilcoxon signed-rank test was used to compare the amount of prescribed patching time and the actual amount of patching achieved prior to the use of elbow splints. This yielded strong evidence $(P \leq 0.001)$ that the amount of patching achieved before the use of splints $(1.5 \pm 1.7 \mathrm{~h})$ was significantly less than the prescribed patching time $(4.95 \pm 1.5 \mathrm{~h})$.

In determining whether the amount of patching time after splints was greater than the patching time before splints, the normality assumption of paired $t$-test was not satisfied. Therefore, a non-parametric Wilcoxon signedrank test was used to compare the amount of patching time achieved before and after the use of elbow splints. There was a strong evidence $(P \leq 0.0001)$ that using elbow splints significantly increased the amount of patching time achieved.

\section{Discussion}

The most common method of amblyopia treatment in children is occlusion therapy. ${ }^{4,5}$ Although it is an easily administered treatment option, the major challenge for its success is the complete reliance on the child's compliance with patching. As compliance with patching is often $<50 \%$ of the prescribed amount of time, ${ }^{5}$ there have been a variety of alternative methods employed to increase patching compliance, with mixed results. Some splinting methods are temporary, but easily removable, such as 
paper towel rolls on the arms, water wings, and hand socks. ${ }^{9}$ Others have used semi-permanent methods of increasing patching compliance, such as suturing the patch to the brow and cheek, using occlusive contact lenses, gluing the fellow eye shut, casting the patient's arms, or even injecting purified botulinum A toxin in to the levator muscle to induce ptosis. ${ }^{5,6,9,10}$ Although these methods may also increase compliance, they are more extreme in nature, can cause pain, increase the risk of infection, and increase the risk of the child developing amblyopia in the fellow eye.

Compared with previously mentioned methods, elbow splints are a safe method of increasing compliance with patching. Elbow splints are easily removed by the parents yet difficult to remove by the children on their own. Even though children were very upset while wearing elbow splints and patch for a mean \pm SD of $91 \pm 116$ min following splint administration, parents found the temporary unhappiness was an acceptable side effect in order to aid in the restoration of vision in the amblyopic eye (Table 2).

These results are important clinically, as it is well established that patching compliance is often $<50 \%$, which makes increasing the VA in the amblyopic eye difficult. ${ }^{5}$ This may be in part, due to the fact that results of consistent patching are not easily visible or instantly obtained, and that patching is typically a long-term strategy. Objective patching compliance measurement tools, comprised of an eye patch with sensors to record when the patch is removed from the skin, ${ }^{11,12}$ could increase the confidence in patching compliance measurement for future studies. These factors may lead to the impact of the patch being minimized the longer the patch is prescribed. If patching compliance is increased through the utilization of elbow splints, the VA in the amblyopic eye can be increased, as seen in the results of this study (Table 1).

This study is limited due to its retrospective nature and consequent challenges in accessing complete data from each patient chart. As well, much of the data, including compliance measures, was garnered through parental report, which may have been influenced by either recall bias or an over-reporting of the effect of elbow splinting. Other limitations of retrospective projects are that inclusion in this study was not based on a randomization model, and that we are unaware of how many parents were offered elbow splints but declined utilization.

\section{Conclusion}

This study provides evidence that in children with amblyopia who have failed occlusion therapy efforts, elbow splints are a viable treatment to be employed in conjunction with occlusion therapy in an attempt to increase compliance rates. Adding elbow splints to the occlusion therapy regimen is an effective and parentally acceptable treatment method to ensure that all is done in order to try and improve vision in amblyopic eyes. Future studies should examine quality of life in patients who are utilizing elbow splints as well as the efficacy of elbow splinting as a method of increasing compliance with occlusion therapy in a randomized fashion. Randomization is necessary in order to definitively determine if this adjunct therapy is a significantly better method of increasing compliance than current common practice.

\section{Summary}

What was known before

- Patching is the cornerstone of pediatric amblyopia treatment, however, compliance with patching has been previously shown to be $<50 \%$.

- There are many different methods that have been tested to increase patching compliance with mixed results.

- If patching compliance is low, visual acuity in the amblyopic eye is less likely to increase.

What this study adds

- This study provides evidence that splinting as a method of increasing patching compliance for amblyopia therapy is a viable treatment.

\section{Conflict of interest}

The authors declare no conflict of interest.

\section{References}

1 Noorden GK. Mechanisms of amblyopia. Adv Ophthalmol 1977; 34: 93-115.

2 Breslin C, Muller C. Vision Loss in Canada 2011. The National Coalition for Vision Health 2011. Available at: http:/ / www.cos-sco.ca/wp-content/uploads/2012/09/ VisionLossinCanada_e.pdf (accessed 20 May 2015).

3 van Leeuwen R, Eijkemans MJ, Vingerling JR, Hofman A, de Jong PT, Simonsz HJ. Risk of bilateral visual impairment in individuals with amblyopia: the Rotterdam study. $\mathrm{Br} J$ Ophthalmol 2007; 91: 1450-1451.

4 The Pediatric Eye Disease Investigator Group. A randomized trial of atropine vs patching for treatment of moderate amblyopia in children. Arch Ophthalmol 2002; 120: 268-278.

5 Wallace MP, Stewart CE, Moseley MJ, Stephens DA, Fielder AR, Monitored Occlusion Treatment Amblyopia Study (MOTAS) Cooperatives et al. Compliance with occlusion therapy for childhood amblyopia. Investig Ophthalmol Vis Sci 2013; 54: 6158-6166.

6 Hiles DA, Galket RJ. Plaster cast arm restraints and amblyopia therapy. J Pediatr Ophthalmol 1974; 11: 151-152.

7 Fralick FB. The treatment of amblyopia. Am J Ophthalmol 1943; 26: 1195-1198. 
8 IBM Corp. Released 2013. IBM SPSS Statistics for Windows, Version 22.0. IBM Corp: Armonk, NY, USA.

9 Rubab S, French D, Levin AV. Glued patches for children resistant to amblyopia occlusion therapy. Arch Ophthalmol 2008; 126: 133-134.

10 Halkiadakis I, Iliaki O, Kalyvianaki M, Tsilimbaris M. Occlusion therapy of unilateral amblyopia with botulinum toxin induced ptosis. Semin Ophthalmol 1995; 79: 585-589.
11 Fielder AR, Irwin M, Auld R, Cocker KD, Jones HS, Moseley MJ. Compliance in amblyopia therapy:

objective monitoring of occlusion. Br J Ophthalmol 1995; 79: 585-589.

12 Simonsz HJ, Polling JR, Voorn R, van Leeuwen J, Meester H, Romijn $\mathrm{C}$ et al. Electronic monitoring of treatment compliance in patching for amblyopia. Strabismus 1999; 7: 113-123. 